\title{
A Role for the Subthalamic Nucleus in Response Inhibition during Conflict
}

\author{
John-Stuart Brittain, ${ }^{1,4}$ Kate E. Watkins, ${ }^{5}$ Raed A. Joundi, ${ }^{1,6}$ Nicola J. Ray, ${ }^{1,6}$ Peter Holland, ${ }^{1,6}$ Alexander L. Green, ${ }^{1,2}$ \\ Tipu Z. Aziz, ${ }^{1,2}$ and Ned Jenkinson ${ }^{1,2,3}$ \\ ${ }^{1}$ Functional Neurosurgery and Experimental Neurology Group, ${ }^{2}$ Nuffield Department of Surgical Sciences, and ${ }^{3}$ Nuffield Department of Clinical \\ Neurosciences, University of Oxford, Oxford OX3 9DU, United Kingdom, ${ }^{4}$ Centre of Excellence in Personalised Healthcare, Institute of Biomedical \\ Engineering, Department of Engineering Science, University of Oxford, Oxford OX3 7DQ, United Kingdom, ${ }^{5}$ Department of Experimental Psychology, \\ University of Oxford, Oxford OX1 3UD, United Kingdom, and 'Department of Physiology, Anatomy and Genetics, University of Oxford, Oxford OX1 3QX, \\ United Kingdom
}

The subthalamic nucleus (STN) is a key node in the network that supports response inhibition. It is suggested that the STN rapidly inhibits basal ganglia activity, to pause motor output during conflict until an appropriate motor plan is ready. Here, we recorded neural activity during a Stroop task from deep brain stimulation electrodes implanted in the human STN. We intended to determine whether cognitive psychological phenomena such as the Stroop effect can be explained via mechanisms of response inhibition involving the STN, or whether higher cognitive centers are alone responsible. We show stimulus-driven desychronization in the beta band (15-35 Hz) that lasts throughout the verbal response, in keeping with the idea that beta-band synchrony decreases to allow motor output to occur. During incongruent trials - in which response times were elongated due to the Stroop effect - a resynchronization was seen in the beta band before response. Crucially, in the incongruent trials during which the participant was unable to withhold the prepotent response, this resynchronization occurred after response onset. We suggest that this beta-band resynchronization pauses the motor system until conflict can be resolved.

\section{Introduction}

Activity in the subthalamic nucleus (STN) suppresses the motor system by inhibiting output from the basal ganglia (Alexander and Crutcher, 1990; Parent and Hazrati, 1995). The STN receives input from the frontal cortex via the hyperdirect pathway (Nambu et al., 2002), such that these executive cortical areas influence STN activity at very short latencies (Maurice et al., 1998; Magill et al., 2004). Because of these properties, it has been suggested, in several models, that the STN lies at the heart of the system controlling response inhibition (Aron and Poldrack, 2006; Frank, 2006; Hikosaka and Isoda, 2010; Munakata et al., 2011). One such model provided computational simulations of the basal ganglia suggesting that cortical conflict signals could generate a temporary delay of motor output via activation of the STN (Frank, 2006). It has since been demonstrated, in line with this model, that interference of the STN compromises the ability

Received May 10, 2012; revised Aug. 3, 2012; accepted Aug. 7, 2012.

Author contributions: J.-S.B., K.E.W., P.H., T.Z.A., and N.J. designed research; J.-S.B., R.A.J., P.H., A.L.G., and N.J. performed research; J.-S.B., K.E.W., N.J.R., and N.J. analyzed data; J.-S.B., K.E.W., and N.J. wrote the paper.

This work was supported by The Centre of Excellence in Personalised Healthcare funded by the Wellcome Trust and Engineering and Physical Sciences Research Council under Grant WT 088877/Z/09/Z. We acknowledge funding from the Medical Research Council, Parkinson's Disease United Kingdom, and the National Institute for Health Research 0xford Biomedical Research Centre. We also thank our two reviewers for their informative and insightful comments.

This article is freely available online through the J Neurosci Open Choice option.

Correspondence should be addressed to John-Stuart Brittain, Charles Wolfson Neuroscience Clinical Research Facility, Level 1, West Wing, John Radcliffe Hospital, 0xford 0X3 9DU, UK. E-mail:john-stuart.brittain@eng.ox.ac.uk. DOI:10.1523/JNEUROSCI.2259-12.2012

Copyright $\odot 2012$ the authors $\quad 0270-6474 / 12 / 3213396-06 \$ 15.00 / 0$ of individuals to delay decision making during high conflict choices (Frank et al., 2007).

Deep brain stimulation (DBS) affords a unique opportunity to stimulate or record the electrical activity of the human STN, the most commonly implanted nucleus for the treatment of Parkinson's disease (PD). As such, response inhibition behavior observed during stimulation of the STN (van den Wildenberg et al., 2006; Ray et al., 2009; Obeso et al., 2011; Swann et al., 2011; Mirabella et al., 2012), and the recording of STN activity during countermanding tasks (Ray et al., 2012), has been used to elucidate the role of the nucleus in response inhibition.

Here, we use the Stroop task in which distractors and attended stimuli are presented simultaneously. This is unlike countermanding paradigms, such as the stop signal task, in which the prepotency of a response is trained by frequent presentation of a cue stimulus with the inhibitory response provided by infrequent presentation of an imperative stop signal. The Stroop task therefore permits evaluation of conflicting cognitive and motor responses, as opposed to explicit reprogramming of the ongoing response.

We hypothesized that functional changes would occur in the beta band (15-35 Hz) because of its association with inhibition in the motor system in diseases such as PD (Brown, 2007; Engel and Fries, 2010; Jenkinson and Brown, 2011) and recent experiments that have demonstrated that induced beta oscillations reduce both the velocity and force rate of movement in healthy humans (Pogosyan et al., 2009; Joundi et al., 2012). More specifically, beta-band activity is known to increase in the STN during "nogo" trials (Kühn et al., 2004) and in the STN and associated right 

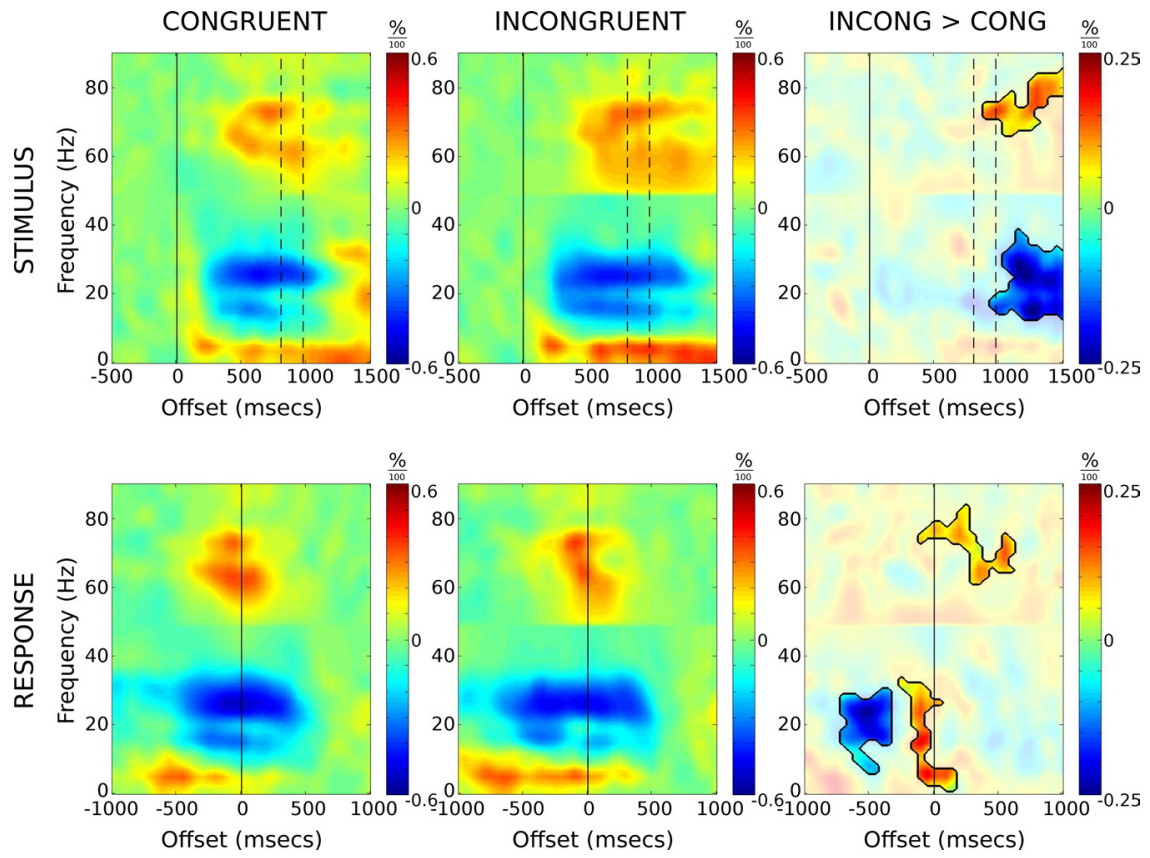

Figure 1. Average time-frequency spectrograms for congruent and incongruent correct response trials, time-locked to stimulus presentation (top row) and verbal response (bottom row). Spectrograms represent power as proportion change values relative to their respective baseline regions. The third column depicts difference spectrograms with incongruent $>$ congruent. Difference spectrograms are masked to highlight regions in which paired $t$ tests remained significant after correction for multiple comparisons (see main text for details). The dashed vertical lines denote mean congruent $(807 \mathrm{~ms}$ ) and incongruent $(980 \mathrm{~ms})$ reaction times. Note the extended beta suppression on incongruent versus congruent trials, and the rebound just before response onset.

inferior frontal cortex during successful stopping in the stop signal task (Swann et al., 2009; Ray et al., 2012). Consistent with the tight relationship between these structures and beta-band activity, neurons in the STN are entrained by the cortex at beta frequencies (Sharott et al., 2009).

\section{Materials and Methods}

Stroop task design followed that of the classic paper (Stroop, 1935), implemented with the colors "RED," "GREEN," "YELLOW," and "BLUE" presented on a black screen $57 \mathrm{~cm}$ in front of the subjects. Words were presented either in their corresponding (congruent) color, or in one of the other three (incongruent) colors. The task requires the subject to suppress the prepotent response (reading the text) and instead respond only with the color of the "ink" that the text appears in. Trials were presented randomly with a 3:2 ratio of incongruent to congruent. Each trial remained on screen for $2.5 \mathrm{~s}$ with a $0.5 \mathrm{~s}$ intertrial interval during which the screen was blank. The task comprised four blocks, each block containing 60 trials. The subjects' verbal responses were recorded using a microphone. Subjects were given a 10 trial practice block (which was repeated until they were comfortable with the task) before testing began. Each experimental session comprised 240 presentations: 96 congruent trials and 144 incongruent trials. The main task was preceded by a control condition (48 trials) during which each of the four words were presented in white text on a black background and participants were required to name the written word (this ensured all patients could read the stimuli used in the subsequent task). Speech records were assessed off-line for fillers (disfluent speech including breaks, irregularities, or nonwords) and errors (responding to the distractor stimulus), both of which were classified as error trials.

The task was performed by 12 patients ( 9 males), 11 of whom had electrodes implanted bilaterally in the STN, and one implanted on the left side only (23 STN recordings in total). All electrode positions were verified by the surgical team with postimplantation MRI scans showing that the electrodes were targeted accurately. Recordings were obtained $4-6 \mathrm{~d}$ after the initial operation, before implantation of the DBS pulse generator. Patients were tested on their usual therapeutic medication in accordance with previous data from PD patients performing Stroop tasks that demonstrated increased impulsivity and error rates when patients were off medication, with a normalization to age-matched control levels when medication was resumed (Djamshidian et al., 2011). All patients were implanted with Medtronic 3389 electrodes, through which local field potentials (LFPs) were recorded, amplified (CED 1902; Cambridge Electronic Design), and digitized at a sampling rate of $2.5 \mathrm{kHz}$ (CED 1401). Data were recorded to hard disk in a bipolar (first spatial derivative) configuration using CED Spike2 software, downsampled $(1 \mathrm{kHz})$, and analyzed off-line using in-house scripts written in Matlab (Mathworks).

To localize our analysis to the electrode contacts within the STN, the contacts with the highest ratio of beta power $(15-35 \mathrm{~Hz})$ to broadband power $(0-100 \mathrm{~Hz})$ were selected. Several studies have demonstrated that increased levels of beta oscillations recorded from individual contacts on the macroelectrode are due to the positioning of those electrode contacts within the STN (Kühn et al., 2005; Weinberger et al., 2006). Indeed, the STN can be localized during surgery through its beta profile (Liu et al., 2001; Chen et al., 2006). One patient failed to demonstrate a clear peak in the beta spectrum and so could not be localized to the STN on either side. This subject was excluded from further analysis.

Data were epoched by time-locking to stimulus presentation ( -500 through $1500 \mathrm{~ms}$ ) and the onset of verbal response ( -1000 through $1000 \mathrm{~ms})$. Epochs were subtracted and variance normalized to baseline periods. Baselines were -500 to $0 \mathrm{~ms}$ for stimulus presentation and 500 to $1000 \mathrm{~ms}$ for responsealigned epochs. Trials with severe artifact were removed ( $8 \pm 4 \%$ of trials on average). Incongruent trials were randomly subsampled to match congruent trial numbers.

Spectrograms were constructed for each trial and averaged per electrode using the set of Hermite functions (Baraniuk and Bayram, 2000) with time-frequency localization parameter $A / 2=5$ (Brittain et al., 2007). Average spectrogram activity reflects both the evoked response (which is phase-locked to the stimulus) and the induced response (which is caused by but not phase-locked to the stimulus). To focus our analysis on the induced oscillatory response, and thus minimize the contribution from transient activity, we additionally computed the spectrogram of the evoked response (event-related potential) per electrode and subtracted this from our average. The resulting spectrograms were convolved using a two-dimensional smoothing operator $(2 \times 2$ unity matrix, equivalent to $100 \mathrm{~ms} \times 1 \mathrm{~Hz}$ ) to aid statistical evaluation. Differences between congruent and incongruent spectrograms were evaluated through pointwise paired $t$ tests over log-spectral values at $p<0.05$ (uncorrected). We corrected for multiple comparisons using permutation testing (1000 iterations) in conjunction with exceedence mass testing at the $\alpha=0.05$ level (Nichols and Holmes, 2002). Briefly, congruent and incongruent spectrograms were randomly reassigned between conditions (per electrode) while maintaining their paired relationship. This procedure was iterated 1000 times to provide a null distribution for statistical testing. Exceedence mass testing involves integrating the excess mass of suprathreshold clusters in the spectrogram and recording the largest per iteration. The top $5 \%$ of this distribution then determined the corrected threshold for imagewise significance.

Average traces across each frequency band of interest were extracted from patient spectrograms (per electrode) and assessed as proportion change values relative to the baseline period as (value - baseline)/baseline. Pointwise paired $t$ tests were applied to proportion change measures between congruent and incongruent trials, with inference on these sta- 
tistics corrected for multiple comparisons using a permutation strategy analogous to that described for spectrograms.

To characterize desynchronization in the beta range, the time course of beta activity (per subject averaged over trials) was smoothed (300 ms) and normalized to the maximum desynchronization trough. Desynchronization and resynchronization latencies were determined as the first and last point at which a half-minimum threshold was crossed. The relationship between trial type (congruent or incongruent), measure (desynchronization latency, resynchronization latency), and side (left vs right hemisphere) were then examined in a three-factor, repeated-measures general linear model (GLM); a Greenhouse-Geisser correction was used to adjust the degrees of freedom when Mauchly's test of sphericity was significant. The Bonferroni correction was used to adjust the $\alpha$ level for post hoc $t$ tests to $p<0.025$ when two between-trial type comparisons were made.

To assess the response during error trials, each correct_incongruent band-limited trace was regenerated for a randomly drawn subsample of epochs (contributing equivalent trial numbers on a subject-by-subject basis to their incorrect_incongruent counterparts). This was repeated 1000 times as part of a bootstrap analysis. The error response was then compared with the resampled population. Of particular interest was the latency of the beta rebound, which was assessed by fitting a Gaussian kernel over the rebound period ( -400 through 150 $\mathrm{ms}$ ).

\section{Results}

\section{Behavioral}

Average median reaction times were longer for incongruent trials $(980 \mathrm{~ms})$ than for congruent $\left(807 \mathrm{~ms} ; t_{(10)}=-8.111 ; p<0.001\right)$. The average median reaction time for reading neutrally colored words (control task) was $503 \mathrm{~ms}$.

Errors were uncommon, contributing on average $1 \pm 3$ (SD) trials per subject (1.3\%) for the congruent condition and $11 \pm 9$ trials $(6.5 \%)$ per subject for the incongruent condition. Only four subjects made errors on the congruent trials.

\section{Frequency modulation}

Spectrogram analysis of the LFP data for congruent and incongruent trials is presented in Figure 1. The spectral content of these data shows regional differences localized to three distinct frequency bands, theta $(5-10 \mathrm{~Hz})$, beta $(15-35 \mathrm{~Hz})$, and gamma $(70-80 \mathrm{~Hz})$. Band-limited traces for each of these frequencies are presented in Figure 2, with pointwise paired $t$ tests providing an indication of the difference between incongruent and congruent trials. Hemispheric differences were examined with no clear bias in response noted across frequency bands (Fig. 2, insets).

Theta $(5-10 \mathrm{~Hz})$ profiles revealed a common pattern of synchronization between task conditions. Upon stimulus presentation, we see a $25 \%$ increase in power, peaking $200 \mathrm{~ms}$ after stimulus presentation followed by a plateau (Fig. 2). Incongruent trials additionally demonstrate a secondary increase in theta power maintaining a higher mean level relative to congruent trials before dropping back to congruent trial levels. Gamma-band activity demonstrated a slow
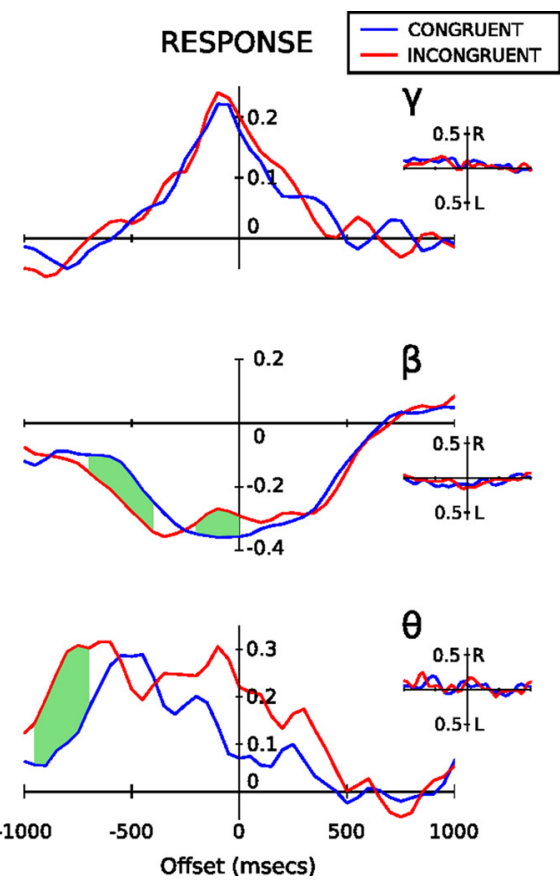

1500 )

$(15-35 \mathrm{~Hz})$, and gamma $(70-80 \mathrm{~Hz})$ presented as proportion change values relative to baseline. The blue lines denote the mean congruent response, and the red lines, the mean incongruent response, for correct response trials only. The dashed lines on the stimulus-locked profiles (left column) represent mean congruent $007 \mathrm{~ms}$ ) and incongruent $(980 \mathrm{~ms})$ reaction times. The green shaded regions represent times where paired $t$ tests demonstrate a ext for details). The insets show hemispheric differences (right- minus left-side response) for congruent (blue) and incongruent (red) trials. Neither congruent nor incongruent results display evidence of statistical separation between sides.

synchronization to peak (at the response time), followed by slow desynchronization back to baseline levels.

Stimulus-locked beta activity showed a pronounced desynchronization $200 \mathrm{~ms}$ after stimulus onset, undergoing a 30\% reduction in resting-level activity (Fig. 2). The time course of beta activity was further explored through a three-factor GLM (see Materials and Methods). There was a significant interaction between trial type and measure $\left(F_{(1,8)}=26.4 ; p<0.005\right)$, but the main effect of, and interactions involving, hemisphere (i.e., left vs right) were not significant. To examine the interaction, left and right hemisphere data were pooled and $t$ tests performed among trial types for the two measures. Analysis revealed that the latency of desynchronization was not dependent upon trial type, but the latency of resynchronization was significantly shorter in congruent compared with incongruent trials $\left(t_{(18)}=-5.099 ; p<\right.$ 0.005). In sum, beta desynchronization occurs at a similar time regardless of trial type or hemisphere, with resynchronization prolonged during incongruent trials in accordance with the longer reaction times observed.

The stimulus-locked response (Fig. 2) reveals prolonged activity in theta, beta, and gamma ranges for incongruent versus congruent trials. The divergence between conditions begins around the time when responses are made to congruent trials and likely reflects a delay in motor response as stimulus conflict is resolved during incongruent trials.

When aligned to verbal response time, a rebound in synchronizing beta activity between -300 and $100 \mathrm{~ms}$ is revealed for incongruent trials, while no such rebound is observed for congruent trials. The rebound in synchronizing beta is transient and begins to desynchronize again immediately before the verbal response is made. The magnitude of peak rebound was significantly 

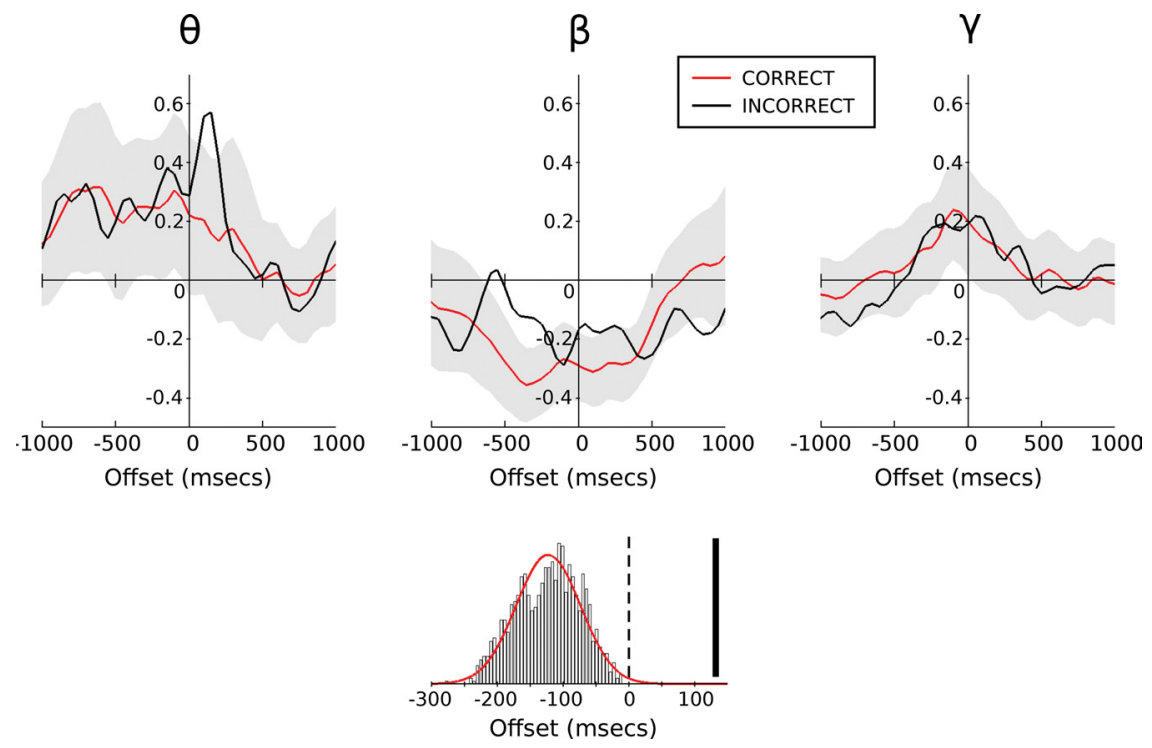

Figure 3. Response-locked change in band-limited power during correct and incorrect incongruent trials relative to baseline (as in Fig. 2). The black lines denote incorrect_incongruent trials with correct_incongruent trials overlaid in red. The shaded regions represent the $95 \%$ population interval derived from bootstrap testing of randomly sampled correct_incongruent trials to match incorrect_incongruent trial numbers. The histogram displays the beta-rebound latency distribution (with Gaussian fit) as determined through bootstrap testing (giving a mean of $-138 \mathrm{~ms}$ ) with incorrect_incongruent rebound latency ( $132 \mathrm{~ms}$ ) represented by the solid vertical bar.

stronger for incongruent trials compared with congruent $\left(t_{(20)}=\right.$ $-4.374 ; p<0.001)$

To address the functional significance of the beta rebound, we compared correct and incorrect trials for the incongruent stimuli (Fig. 3). Both correct and incorrect incongruent trials demonstrate a beta rebound after the initial desynchronization. However, it is notable that the beta rebound in correct trials occurs before the response time, whereas for incorrect trials the rebound peaks after the verbal response has been made. Through bootstrap analysis, we see that the latency of beta rebound associated with an incorrect response is much later than the latency observed on correct trials (132 vs $-138 \mathrm{~ms}$ aligned to verbal response onset; Fig. 3 ), producing a $z$ score of 5.25 ( $p<0.0001$, two-tailed test). Importantly, all rebound latencies observed for correct responses occurred before the verbal response time, whereas the rebound associated with incorrect responses occurred after the onset of response.

Bootstrap analysis also revealed a late peak in theta-band activity during error trials (Fig. 3). This theta-band activity peaked around $150 \mathrm{~ms}$ after verbal response onset.

\section{Discussion}

Here, we show a specific rebound in beta-band activity in the STN in response to incongruent Stroop stimuli. Increased beta is related to an akinetic state in Parkinson's disease (Jenkinson and Brown, 2011), and induction of beta oscillations reduces both the velocity and force rate of movement in healthy humans (Pogosyan et al., 2009; Joundi et al., 2012). We deduce, therefore, that the beta rebound delays the prepotent response, so that the conflicting stimuli can be resolved and the correct response given. Our finding is consistent with the role of the STN in contemporary models of response inhibition during conflict (Frank, 2006; Aron, 2007). This position is further supported by the fact that the timing of the beta rebound in error trials occurs later, at a time after which the prepotent response has already been given. This is one of the first reports of electrophysiological evidence from humans that cognitive interference phenomena such as the Stroop effect may be-at least in part—mediated through the same mechanisms as response inhibition during simple motor tasks (e.g., stop signal task, go/no-go).

The consistency of the timing of betaband desynchronization within the STN across task conditions suggests a common role in motor preparation, regardless of conflict, or eventual reaction time. A prolongation in the duration of beta desynchronization in the incongruent condition (relative to congruent) can be explained by a delayed response in this condition. However, the consistency in timing of beta desynchronization also suggests that the motor system is primed to respond before conflict is resolved during incongruent trials, requiring inhibition before correct response selection. Specifically, correct responses to incongruent trials were associated with a beta rebound occurring before response, whereas incorrect responses were associated with a rebound occurring after the onset of response. This is consistent with a model of response inhibition in which the STN acts to delay the motor action, with the beta rebound providing a signature of

this activity.

While beta desynchronization has been widely reported during various forms of volitional movement, its relationship with speech production is less well described. Here, we replicate findings showing similar beta desynchronization before and during speech production (Hebb et al., 2012). Furthermore, we demonstrate increased power in the gamma and theta bands consistent with the only other report of STN activity during speech (Anzak et al., 2011).

Conflict and inhibition in the motor system are often explored within the context of stop signal paradigms. During the stop signal task, a series of attended (go signal) stimuli are presented, and on a minority of trials the sudden presentation of an inhibitory stimulus (the stop signal) alerts the participant to withhold their response. Recent electrophysiological evidence obtained during a stop signal task (Swann et al., 2009) demonstrated exaggerated beta activity on successful versus unsuccessful stop trials over right inferior frontal gyrus. This was accompanied by reduced desynchronization in the broader $8-30 \mathrm{~Hz}$ range over primary motor cortex. Further evidence using the stop signal task has more recently been obtained by direct electrophysiological recordings from the STN (Ray et al., 2012), where the timing of beta rebound was shown to correlate with the stop signal reaction time. Similar beta activity in our study (Fig. 3) underscores again the possibility of a common neural mechanism for various forms of response inhibition including spoken responses (Xue et al., 2008). Xue et al. (2008), however, demonstrated activation of the STN during response inhibition in a manual task but failed to demonstrate the same modulation during a verbal response. This failure may be due in part to difficulties in localizing fMRI blood oxygen level-dependent activity in the STN, especially during speech production. Here, our use of direct electrophysiological recording has allowed us to demonstrate a clear LFP modulation to incongruent stimuli in STN during a task requiring a verbal response. 
Our observed persistent theta response during incongruent trials is also consistent with previous studies showing raised theta activity during high conflict scenarios, both in mediofrontal EEG (Cavanagh et al., 2011) and in the STN (Cavanagh et al., 2011; Fumagalli et al., 2011). Cavanagh proposes that this theta activity is predictive of the subjects' tendency to delay responding to improve accuracy. Cavanagh et al. (2011) also demonstrated increased mediofrontal theta power (and phase synchrony) in response to errors on a probabilistic reinforcement task, reminiscent of the theta peak observed on error trials in our task.

While our results remain consistent with a number of theories on the role the STN plays in conflict-induced response inhibition (Aron et al., 2007; Frank et al., 2007), it should be noted that our recordings are limited to the STN. We cannot rule out, therefore, a more global beta response arising in other basal ganglia nuclei, as recently observed in the rat (Leventhal et al., 2012). Further investigations of response inhibition in other DBS targets within the human corticobasal ganglia system would elucidate whether the changes described here are exclusive to the STN.

Our results demonstrate that the motor system is primed upon presentation of the stimulus regardless of task condition. The common beta desynchronization response to all stimuli presentations, regardless of congruence, strongly suggests that, during the Stroop task, we prepare a motor response before the distractor conflict is resolved. In a motor planning task, Tzagarakis et al. (2010) found that the magnitude of peri-Rolandic magnetoencephalographic beta desynchronization correlated with response certainty, in their case, the likelihood of radial cues for joystick movement. Likewise, recent microelectrode recordings from the STN of PD patients demonstrated an increase in firing rates in high- versus low-conflict trials during a probabilistic learning task (Zaghloul et al., 2012). The initial beta desynchronization we observed during incongruent trials might, therefore, reflect initial response certainty, followed by a rebound in beta activity upon detection of conflict. We suggest that this beta activity rebound seen during conflict is an inhibitory signal via the hyperdirect pathway to pause action selection, enabling a choice response to be made between color and word. This mechanism would also explain the delayed rebound observed in error trials. In these trials, the inhibitory signal arrives too late to pause action selection before the conflict is resolved resulting in incorrect responses (Fig. 3, compare red and black traces).

We conclude that modulation of power in beta-band activity in the STN is critical in the processing of action selection and response during the presentation of conflicting stimuli. Specifically, beta power has to be reduced during the response. In incongruent trials with conflict, beta activity is momentarily increased to delay the response, so that conflict can be resolved and the correct response selected. We suggest that this transitory increase in beta-band power in the STN is the neural signature of the hyperdirect inhibitory signal carried from the cortex to the output of the basal ganglia via the STN. Furthermore, we suggest that this is a common mechanism for response inhibition in both the cognitive and motor systems.

\section{References}

Alexander GE, Crutcher MD (1990) Functional architecture of basal ganglia circuits: neural substrates of parallel processing. Trends Neurosci $13: 266-271$

Anzak A, Gaynor L, Beigi M, Limousin P, Hariz M, Zrinzo L, Foltynie T, Brown P, Jahanshahi M (2011) A gamma band specific role of the subthalamic nucleus in switching during verbal fluency tasks in Parkinson's disease. Exp Neurol 232:136-142.
Aron AR (2007) The neural basis of inhibition in cognitive control. Neuroscientist 13:214-228.

Aron AR, Poldrack RA (2006) Cortical and subcortical contributions to Stop signal response inhibition: role of the subthalamic nucleus. J Neurosci 26:2424-2433.

Aron AR, Behrens TE, Smith S, Frank MJ, Poldrack RA (2007) Triangulating a cognitive control network using diffusion-weighted magnetic resonance imaging (MRI) and functional MRI. J Neurosci 27:3743-3752.

Baraniuk RG, Bayram M (2000) Multiple window time varying spectrum estimation. Cambridge, UK: Cambridge UP.

Brittain JS, Halliday DM, Conway BA, Nielsen JB (2007) Single-trial multiwavelet coherence in application to neurophysiological time series. IEEE Trans Biomed Eng 54:854-862.

Brown P (2007) Abnormal oscillatory synchronisation in the motor system leads to impaired movement. Curr Opin Neurobiol 17:656-664.

Cavanagh JF, Wiecki TV, Cohen MX, Figueroa CM, Samanta J, Sherman SJ, Frank MJ (2011) Subthalamic nucleus stimulation reverses mediofrontal influence over decision threshold. Nat Neurosci 14:1462-1467.

Chen CC, Pogosyan A, Zrinzo LU, Tisch S, Limousin P, Ashkan K, Yousry T, Hariz MI, Brown P (2006) Intra-operative recordings of local field potentials can help localize the subthalamic nucleus in Parkinson's disease surgery. Exp Neurol 198:214-221.

Djamshidian A, O'Sullivan SS, Lees A, Averbeck BB (2011) Stroop test performance in impulsive and non impulsive patients with Parkinson's disease. Parkinsonism Relat Disord 17:212-214.

Engel AK, Fries P (2010) Beta-band oscillations-signalling the status quo? Curr Opin Neurobiol 20:156-165.

Frank MJ (2006) Hold your horses: a dynamic computational role for the subthalamic nucleus in decision making. Neural Netw 19:1120-1136.

Frank MJ, Samanta J, Moustafa AA, Sherman SJ (2007) Hold your horses: impulsivity, deep brain stimulation, and medication in parkinsonism. Science 318:1309-1312.

Fumagalli M, Giannicola G, Rosa M, Marceglia S, Lucchiari C, Mrakic-Sposta S, Servello D, Pacchetti C, Porta M, Sassi M, Zangaglia R, Franzini A, Albanese A, Romito L, Piacentini S, Zago S, Pravettoni G, Barbieri S, Priori A (2011) Conflict-dependent dynamic of subthalamic nucleus oscillations during moral decisions. Soc Neurosci 6:243-256.

Hebb AO, Darvas F, Miller KJ (2012) Transient and state modulation of beta power in human subthalamic nucleus during speech production and finger movement. Neuroscience 202:218-233.

Hikosaka O, Isoda M (2010) Switching from automatic to controlled behavior: cortico-basal ganglia mechanisms. Trends Cogn Sci 14:154-161.

Jenkinson N, Brown P (2011) New insights into the relationship between dopamine, beta oscillations and motor function. Trends Neurosci 34:611-618.

Joundi RA, Jenkinson N, Brittain JS, Aziz TZ, Brown P (2012) Driving oscillatory activity in the human cortex enhances motor performance. Curr Biol 22:403-407.

Kühn AA, Williams D, Kupsch A, Limousin P, Hariz M, Schneider GH, Yarrow K, Brown P (2004) Event-related beta desynchronization in human subthalamic nucleus correlates with motor performance. Brain 127:735-746.

Kühn AA, Trottenberg T, Kivi A, Kupsch A, Schneider GH, Brown P (2005) The relationship between local field potential and neuronal discharge in the subthalamic nucleus of patients with Parkinson's disease. Exp Neurol 194:212-220.

Leventhal DK, Gage GJ, Schmidt R, Pettibone JR, Case AC, Berke JD (2012) Basal ganglia beta oscillations accompany cue utilization. Neuron 73:523-536.

Liu X, Rowe J, Nandi D, Hayward G, Parkin S, Stein J, Aziz T (2001) Localisation of the subthalamic nucleus using Radionics Image Fusion and Stereoplan combined with field potential recording. A technical note. Stereotact Funct Neurosurg 76:63-73.

Magill PJ, Sharott A, Bevan MD, Brown P, Bolam JP (2004) Synchronous unit activity and local field potentials evoked in the subthalamic nucleus by cortical stimulation. J Neurophysiol 92:700-714.

Maurice N, Deniau JM, Glowinski J, Thierry AM (1998) Relationships between the prefrontal cortex and the basal ganglia in the rat: physiology of the corticosubthalamic circuits. J Neurosci 18:9539-9546.

Mirabella G, Iaconelli S, Romanelli P, Modugno N, Lena F, Manfredi M, Cantore G (2012) Deep brain stimulation of subthalamic nuclei affects 
arm response inhibition in Parkinson's patients. Cereb Cortex 22:1124-1132.

Munakata Y, Herd SA, Chatham CH, Depue BE, Banich MT, O'Reilly RC (2011) A unified framework for inhibitory control. Trends Cogn Sci 15:453-459.

Nambu A, Tokuno H, Takada M (2002) Functional significance of the cortico-subthalamo-pallidal "hyperdirect" pathway. Neurosci Res 43: $111-117$.

Nichols TE, Holmes AP (2002) Nonparametric permutation tests for functional neuroimaging: a primer with examples. Hum Brain Mapp 15:1-25.

Obeso I, Wilkinson L, Casabona E, Bringas ML, Álvarez M, Álvarez L, Pavón N, Rodríguez-Oroz MC, Macías R, Obeso JA, Jahanshahi M (2011) Deficits in inhibitory control and conflict resolution on cognitive and motor tasks in Parkinson's disease. Exp Brain Res 212:371-384.

Parent A, Hazrati LN (1995) Functional anatomy of the basal ganglia. I. The cortico-basal ganglia-thalamo-cortical loop. Brain Res Rev 20:91-127.

Pogosyan A, Gaynor LD, Eusebio A, Brown P (2009) Boosting cortical activity at beta band frequencies slows movement in humans. Curr Biol 19:1637-1641.

Ray NJ, Jenkinson N, Brittain J, Holland P, Joint C, Nandi D, Bain PG, Yousif N, Green A, Stein JS, Aziz TZ (2009) The role of the subthalamic nucleus in response inhibition: evidence from deep brain stimulation for Parkinson's disease. Neuropsychologia 47:2828-2834.

Ray NJ, Brittain JS, Holland P, Joundi RA, Stein JF, Aziz TZ, Jenkinson N (2012) The role of the subthalamic nucleus in response inhibition: evidence from local field potential recordings in the human subthalamic nucleus. Neuroimage 60:271-278.

Sharott A, Moll CK, Engler G, Denker M, Grün S, Engel AK (2009) Different subtypes of striatal neurons are selectively modulated by cortical oscillations. J Neurosci 29:4571-4585.

Stroop JR (1935) Studies of interference in serial verbal reactions. J Exp Psychol 18:643-662.

Swann N, Tandon N, Canolty R, Ellmore TM, McEvoy LK, Dreyer S, DiSano M, Aron AR (2009) Intracranial EEG reveals a time- and frequencyspecific role for the right inferior frontal gyrus and primary motor cortex in stopping initiated responses. J Neurosci 29:12675-12685.

Swann N, Poizner H, Houser M, Gould S, Greenhouse I, Cai W, Strunk J, George J, Aron AR (2011) Deep brain stimulation of the subthalamic nucleus alters the cortical profile of response inhibition in the beta frequency band: a scalp EEG study in Parkinson's disease. J Neurosci 31:5721-5729.

Tzagarakis C, Ince NF, Leuthold AC, Pellizzer G (2010) Beta-band activity during motor planning reflects response uncertainty. J Neurosci 30:11270-11277.

van den Wildenberg WP, van Boxtel GJ, van der Molen MW, Bosch DA, Speelman JD, Brunia CH (2006) Stimulation of the subthalamic region facilitates the selection and inhibition of motor responses in Parkinson's disease. J Cogn Neurosci 18:626-636.

Weinberger M, Mahant N, Hutchison WD, Lozano AM, Moro E, Hodaie M, Lang AE, Dostrovsky JO (2006) Beta oscillatory activity in the subthalamic nucleus and its relation to dopaminergic response in Parkinson's disease. J Neurophysiol 96:3248-3256.

Xue G, Aron AR, Poldrack RA (2008) Common neural substrates for inhibition of spoken and manual responses. Cereb Cortex 18:1923-1932.

Zaghloul KA, Weidemann CT, Lega BC, Jaggi JL, Baltuch GH, Kahana MJ (2012) Neuronal activity in the human subthalamic nucleus encodes decision conflict during action selection. J Neurosci 32:2453-2460. 